\title{
A short geostatistical study of the three-dimensional spatial structure of fumonisins in stored maize
}

\author{
M. Rivas Casado ${ }^{a^{*}}$, D.J. Parsons ${ }^{a}$, N. Magan ${ }^{a}$, R.M. Weightman ${ }^{b}$, P Battilani $^{c}$, A Pietri $^{c}$ \\ ${ }^{a}$ Cranfield University, Cranfield, Bedford, MK43 OAL, UK \\ ${ }^{b}$ Centre for Sustainable Crop Management, ADAS UK Ltd, Boxworth, Cambridge CB23 \\ $4 N N$, UK. ${ }^{\mathrm{c}}$ Università Cattolica del Sacro Cuore, via Emilia Parmense 84, 29100 Piacenza, \\ Italy \\ *Corresponding author. Email: m.rivas-casado@cranfield.ac.uk
}

\begin{abstract}
The heterogeneous three-dimensional spatial distribution of mycotoxins has been proven to be one of the main limitations for the design of effective sampling protocols. Current sampling collection protocols for mycotoxins have been designed to estimate the mean concentration and fail to characterise the spatial distribution of the mycotoxin concentration due to the aggregation of the incremental samples. Geostatistical techniques have been successfully applied to overcome similar problems in many research areas. However, little work has been developed on the use of geostatistics for the design of sampling protocols for mycotoxins. This paper focuses on the analysis of the two and three-dimensional spatial structure of fumonisins $\mathrm{B}_{1}\left(\mathrm{FB}_{1}\right)$ and $\mathrm{B}_{2}\left(\mathrm{FB}_{2}\right)$ in maize in a bulk store using a geostatistical approach and on how results inform on determining the number and location of incremental samples to be collected. The spatial correlation between $\mathrm{FB}_{1}$ and $\mathrm{FB}_{2}$, as well as between the number of kernels infected and the level of contamination was investigated. For this purpose, a bed of maize was sampled at different depths to generate a unique three-dimensional data set of $\mathrm{FB}_{1}$ and $\mathrm{FB}_{2}$. The analysis found no clear evidence of spatial structure in either the two-dimensional or three-dimensional analyses. The number of Fusarium infected kernels was not a good indicator for the prediction of fumonisin concentration and there was no spatial correlation between the concentrations of the two fumonisins.
\end{abstract}

\section{Keywords}

cereals, fungi, Fusarium, mycotoxins, distribution

$\begin{array}{ll}\text { Journal } & \text { World Mycotoxin Journal } \\ \text { Publisher } & \text { Wageningen Academic Publishers } \\ \text { ISSN } & \text { 1875-0710 (Print) 1875-0796 (Online) } \\ \text { Issue } & \text { Volume 3, Number 1 / February 2010 } \\ \text { DOI } & 10.3920 / \text { WMJ2009.1178 } \\ \text { Pages } & 95-103 \\ \text { Online Date } & \text { Tuesday, February 09, 2010 }\end{array}$




\section{Introduction}

To reduce consumer exposure to mycotoxins it is critical to design sampling protocols that ensure representative samples for analysis. Regulations on how to sample for target mycotoxins and which levels are acceptable for consumption have already been established (European Commission, 2006a, 2006b, 2007). The proposed sampling requires the collection of several incremental samples (i.e. small quantities of the commodity) at different locations. These are mixed to form the aggregate sample. If this is large, it is mixed and subsampled to form the laboratory sample for analysis, otherwise the full aggregate sample is used. However, this type of sampling is intended only to estimate the mean concentration and is unable to characterise the variability or the spatial distribution due to the aggregation of the increments.

The heterogeneous distribution of mycotoxins in space has been recognised to be one of the main limitations for the design of effective sampling protocols (Stroka et al., 2004, Schatzki, 1995a and 1995b, Jewers et al., 1988 and Battilani et al., 2006). Several studies have looked into this limitation in more detail. For example: Johansson et al. (2006) looked at the spatial distribution of fumonisin and aflatoxin concentration in maize; Whitaker (2003) investigated the sources of error in the mycotoxin test procedure for aflatoxin in raw shelled peanuts; Coker et al. (1995) reviewed the complexities associated with the design of sampling plans; Maanen and $\mathrm{Xu}$ (2003) identified the difficulties of sampling the spatial aggregation of plant disease; Macarthur et al. (2006) identified the need to account for the spatial heterogeneity of mycotoxins to design adequate sampling programmes; and Whitaker (2006) identified the need for methods that focus on defining the number of samples required to obtain a specific level of confidence on the mean mycotoxin concentration and on determining the location of the samples to be taken.

There has been some interest in characterising the two-dimensional spatial heterogeneity of mycotoxins to determine the number and location of incremental samples in sampling protocols. For example, Rivas et al. (2009a and 2009b) used geostatistics to model the twodimensional spatial structure of ochratoxin A (OTA) and deoxynivalenol (DON) in bulk commodities and Rekah et al. (1999) used it for Fusarium crown and root rot in tomatoes. However, there has been much less effort on the design of sample collection protocols that account for their three-dimensional spatial heterogeneity. Johansson et al. (2006) looked at the three-dimensional distribution of fumonisin and aflatoxin in maize commodities by investigating the contamination levels in damaged kernels, whole kernels and other materials (i.e. broken and foreign material). They found that toxins were concentrated in the poor quality components within stores of maize. These correspond to the smaller size particles and generally deposit at the bottom of the commodity. However, the study did not statistically quantify the three-dimensional spatial heterogeneity. This could be achieved applying geostatistics. Three-dimensional geostatistics has been successfully applied to reconstruct the large to intermediate heterogeneity of aquifers (Falivene et al., 2007), to reproduce the spatial variability of rock mass quality for geological excavations (Stavropoulou et al., 2007) and to map three-dimensional water-quality data to better protect the future health of the Chesapeake Bay and its tidal tributary system (Chehata et al., 2007).

Geostatistics can also be used to investigate the spatial autocorrelation between variables. The high cost associated to sample collection and analysis usually results in a reduced number of incremental samples being taken from a commodity. This has an impact on the accuracy of the estimated mean concentration. Further understanding of the spatial 
correlation between variables will inform on how the mycotoxin concentration can be estimated using variables that are less costly and time consuming. For example, the number of kernels infected could be used for this purpose. Reid et al. (1996) looked at the correlation between the mean disease severity rating and the percentage of symptomatic kernels for Fusarium graminearum inoculated maize ears. Results showed that there was a positive association between the two variables. Desjardins et al. (1998) found that the highest levels of Fusarium moniliforme were in the symptomatic kernels. Uhlig et al. (2004) looked at the relationship between the number of kernels infected with Fusaria and the moniliformin fungal methabolite in Norwegian grain samples of oats, barley and wheat. However, none of these studies have looked at how spatial statistical analysis could improve the understanding of the correlation between variables.

This paper focuses on the analysis of the two and three-dimensional spatial structure of $\mathrm{FB}_{1}$ and $\mathrm{FB}_{2}$ in stored maize using a geostatistical approach. The spatial correlation between $\mathrm{FB}_{1}$ and $\mathrm{FB}_{2}$, as well as between the number of kernels infected and the level of contamination were also characterised using geostatistical techniques. For this purpose, a bed of maize was sampled at different depths to generate a unique three-dimensional data set of $\mathrm{FB}_{1}$ and $\mathrm{FB}_{2}$. This is the first study that tries to quantify the three-dimensional spatial structure of mycotoxins post-harvest. The analysis investigated the following hypotheses:

- The fumonisin concentrations have spatial structures that can be quantified using geostatistics.

- The concentration of fumonisin is related to the number of infected kernels

- The concentration of fumonisins $\mathrm{B}_{1}$ and $\mathrm{B}_{2}$ are spatially correlated.

\section{Materials and Methods}

\section{Data collection}

Data were collected from a bed of maize in a large store with an area of approximately $3800 \mathrm{~m}^{2}$ and a height of approximately $10 \mathrm{~m}$. The kernels, which came from several fields, were stored against the walls, leaving the central space clear. The samples were taken in three planar layers inclined at $45^{\circ}$, parallel to the open face of the bed, providing a threedimensional grid of points with 10 (horizontal) by 5 (vertical) points in each layer at $0.5 \mathrm{~m}$ spacing (Figure 1). The samples were taken using a specially designed probe which was inserted horizontally through the face. The probe had a conical chamber at the tip that could be opened and closed using a lever at the end of the shaft (Figure 2), collecting about $200 \mathrm{~g}$ of grain. A metal frame $0.5 \mathrm{~m}$ square was placed on the face and used to locate the insertion points, and the three samples from different layers at each point were taken before moving the frame to minimise disturbance of the grain.

\section{Fusarium isolation and analyses of fumonisins}

The percentage of kernels infected by Fusarium section Liseola was also determined. Fifty kernels were randomly selected from each sample, surface disinfected with a solution of $1 \%$ sodium hypochlorite and $90 \%$ ethyl alcohol for 2 min and rinsed with sterile distilled water. The kernels were plated in Petri dishes on Potato Dextrose Agar containing streptomycin (PDA: potato broth $600 \mathrm{~g}$, sucrose $10 \mathrm{~g}$, agar $15 \mathrm{~g}$, water to $1 \mathrm{~L}$ ) and incubated at $25^{\circ} \mathrm{C}$ for 7 days. Colonies which resembled Fusaria, were transferred to new Petri dishes of the same medium, incubated for 7-14 days and identified according to Burgess (1994). The number of kernels with Fusarium verticillioides and F.proliferatum were counted. 


\section{Fumonisin analyses}

The $\mathrm{FB}_{1}$ and $\mathrm{FB}_{2}$ contents were analyzed according to the method proposed by Visconti et al. (2001), with some modifications. After grinding the entire sample to pass a $1 \mathrm{~mm}$ sieve and homogenization, fumonisins were extracted from $20 \mathrm{~g}$ of meal in a plastic centrifuge bottle with $100 \mathrm{ml}$ of acetonitrile:methanol:water $(25: 25: 50, \mathrm{v} / \mathrm{v} / \mathrm{v})$. After extraction for $45 \mathrm{~min}$ using a rotary-shaking stirrer and centrifugation at $4500 \times \mathrm{x}$ for $6 \mathrm{~min}$, the supernatant was poured into a flask; another $100 \mathrm{ml}$ of the same solution was added to the residue in the centrifuge bottle, and a second extraction performed for $30 \mathrm{~min}$. The combined extracts were filtered through a folded filter-paper. An aliquot of $2 \mathrm{ml}$ was diluted with $20 \mathrm{ml}$ of $0.1 \mathrm{M}$ phosphate buffered saline (PBS, pH 7.4) and purified through an immunoaffinity column (RBiopharm Rhône Ltd., Glasgow, Scotland, UK); after washing the column with PBS (2 ml), the fumonisins were slowly eluted $\left(0.5 \mathrm{ml} \mathrm{min}^{-1}\right)$ with methanol $(6 \mathrm{ml})$ into a graduated glass vial; subsequently, the eluate was concentrated to $2 \mathrm{ml}$ under a gentle stream of nitrogen. Analysis was carried out using a LC-MS/MS system, (Thermo-Fisher Scientific, San Jose, CA, USA), as previously described (Battilani et al., 2008). The limit of detection (LOD, signal-to-noise ratio of 3:1) and of quantification (LOQ, signal-to-noise ratio of 10:1) were respectively 10 and $30 \mu \mathrm{g} \mathrm{kg}^{-1}$. Recovery percentages were evaluated by spiking known blank samples. Average recovery values were: $95.5 \pm 1.9 \%$ for $\mathrm{FB}_{1}$ and $93.6 \pm 2.1 \%$ for $\mathrm{FB}_{2}$. The results of the analyses were not corrected for recovery.

Five primary samples, with different fumonisin levels, were re-analyzed two more times to estimate the precision of the analysis, which proved the differences between measurements on the same sample to be smaller than $5 \%$.

\section{Data analysis}

Two dimensional geostatistical analysis was conducted to investigate the spatial structure within each layer. Geostatistical analysis is based on the semi-variogram, a plot that relates the distance between any two points with the semi-variance (Figure 3). The semi-variance is a measure of the similarity between any two points; the closer two points are the smaller their semi-variance and the larger their spatial autocorrelation. The semi-variance is calculated as follows:

$\hat{\gamma}(h)=\frac{1}{2 m(h)} \sum_{i=1}^{m(h)}\left(z\left(x_{i}\right)-z\left(x_{i}+h\right)\right)^{2}$

where $z\left(x_{i}\right)$ is the value of variable $z$ at location $x_{i}, m(h)$ is the number of pairs of data points separated by distance $h$ and $\gamma(h)$ is the average semi-variance of all pairs of data points separated by distance $h$, which is known as the lag distance or lag. The semi-variance for a given lag distance is estimated by using all the points separated by distances within a certain tolerance of the required lag distance. The resulting interval is called the lag tolerance. The graph of semi-variance against lag distance is the experimental semi-variogram. The model fitted to the experimental semi-variogram is the empirical semi-variogram.

The empirical semi-variogram is described using three parameters: the sill, the range and the nugget (Figure 3 ). The sill is the a priori variance, $\sigma^{2}$, of the process and is generally assumed to be equal to the variance of the population (Barnes, 1991). The range is the 
distance at which pairs of points are spatially independent and lack autocorrelation. The nugget is the semi-variance at a lag distance of 0 , and identifies the measurement error and the variations that occur over lag distances less than the shortest sampling interval (Webster and Oliver, 2000).

Geostatistical analysis is more effective when the variable has a normal (Gaussian) distribution and does not present any trend. Values of skewness and kurtosis were calculated to test for normality. Where the test failed a Box-Cox transformation (Equation 2) was applied:

$$
\tau(X)=\left(X^{\lambda}-1\right) / \lambda
$$

where $X$ is the variable to be transformed, $\tau(X)$ is the Box-Cox transformed variable and the parameter $\lambda$ is a constant derived iteratively from the data. Regression analysis was used to test for linear or quadratic trends in the $\mathrm{X}$ and $\mathrm{Y}$ directions (Figure 1). Data were assumed to have trend when the variance accounted for by the fitted model was larger than $20 \%$.

For each layer, the experimental semi-variograms were derived in the $\mathrm{X}$ and $\mathrm{Y}$ directions to test for differences in the spatial pattern. It was found that there were insufficient points in the $\mathrm{Y}$ direction to give a reliable semi-variogram, so the data were assumed to be isotropic (no change in the semi-variogram parameters due to direction) and the averaged semi-variogram for all the directions, also known as the omnidirectional semi-variogram, was calculated. The maximum distance for the semi-variogram calculation was set to $2.5 \mathrm{~m}$, because this was the point at which the number of pairs of data points started to decrease. The lag tolerance was set to $0.1 \mathrm{~m}$, because larger values did not provide enough points to fit the semi-variogram model.

The three-dimensional spatial structure was characterised using a three-dimensional semivariogram calculated from the combined data set. The three-dimensional semi-variogram was calculated for $\mathrm{FB}_{1}$ and $\mathrm{FB}_{2}$ for the same values of maximum distance and lag tolerance used in the two-dimensional semi-variogram calculations. The spherical (Equation 3) and exponential (Equation 4) empirical semi-variogram models were fitted using least squares:

$$
\begin{aligned}
& \hat{\gamma}(h)= \begin{cases}c_{0}+c\left(\frac{3 h}{2 a}-\frac{1}{2}\left(\frac{h}{a}\right)^{3}\right) & \text { for } h \leq a \\
c_{0}+c & \text { for } h>a\end{cases} \\
& \hat{\gamma}(h)= \begin{cases}c_{0}+c(1-\exp (h / r)) & \text { for } h \leq a \\
c_{0}+r & \text { for } h>a\end{cases}
\end{aligned}
$$

where $c_{o}$ is the nugget, $c$ is the sill, $a$ is the range, $\mathrm{r}$ is the effective range and $h$ is the lag distance. The exponential model approaches its sill asymptotically and therefore, does not have a finite range. The effective range $r$ is calculated instead and is assumed to be approximately $3 a$. The model with the best fit was selected.

Cross-variograms were used to investigate whether the number of kernels infected was related to the concentration of the fumonisins within each layer and for the full data set. The cross-variogram provided information on their spatial correlation. The cross-variogram, that 
is the plot of semi-variance between two variables against lag distance, $\Gamma(h)$ is calculated as follows (Nielsen and Wendroth, 2003).

$$
\Gamma(h)=\frac{1}{2 N(h)} \sum_{i=1}^{N(h)}\left[A_{i}\left(x_{i}\right)-A_{i}\left(x_{i}+h\right)\right]\left[B_{i}\left(x_{i}\right)-B_{i}\left(x_{i}+h\right)\right]
$$

where $h$ is the distance between sampling locations, $A$ and $B$ are two variables and $N$ is the total number of measurements taken. The cross-variogram was also calculated to assess the spatial correlation of fumonisin $\mathrm{B}_{1}$ with $\mathrm{B}_{2}$. Positive cross-variogram values indicate that the two variables vary jointly in space, negative values show variables that vary in opposite spatial directions and null cross-variogram represent variables that vary independently in space (Nielsen and Wendroth, 2003).

\section{Results}

The $\mathrm{FB}_{1}$ and $\mathrm{FB}_{2}$ data sets did not follow Normal distributions. Box-Cox transformations with $\lambda$ value equal to 0.219273 for $\mathrm{FB}_{1}$ and 0.089097 for $\mathrm{FB}_{2}$ were carried out. This guaranteed the Normality of the data in each layer and in whole data set. Table 1 summarises the descriptive statistics for the raw data set and the results for the Box-Cox transformed data are in Table 2. The trend model accounted for less than $20 \%$ of the variance so the data sets were not de-trended. The infected kernel count data was log transformed to meet the normality requirement.

Similar patterns were evident for $\mathrm{FB}_{1}$ and $\mathrm{FB}_{2}$ within each layer, with the peak concentrations occurring at the same locations (Figure 4). However, there was no obvious correspondence between the patterns in different layers. There was insufficient information to conclude whether there was a trend with distance into the bed.

For each layer the concentration of $\mathrm{FB}_{1}$ was larger than $\mathrm{FB}_{2}$. Layer 1 showed the largest mean and maximum contamination levels for both $\mathrm{FB}_{1}$ and $\mathrm{FB}_{2}$, followed by layer 2 and layer 3 and the ratio $\mathrm{FB}_{2} / \mathrm{FB}_{1}$ was $0.78,0.70$ and 0.57 , respectively. Both skewness and kurtosis excess were close to 0 after the transformation (Table 2). A better approximation to Normality could have been obtained by removing the extreme values, but this would have changed the spatial structure by removing the peak values, so no outliers or extreme values were removed. 
Table 1. Descriptive statistics by layer of data collected

\begin{tabular}{|c|c|c|c|c|c|}
\hline Mycotoxin & Statistic & Layer 1 & Layer 2 & Layer 3 & Combined \\
\hline \multirow{10}{*}{$\mathrm{FB}_{1}$} & Number of points & 50 & 50 & 50 & 150 \\
\hline & Min, $\mu \mathrm{g} / \mathrm{kg}$ & 417 & 191 & 159 & 159 \\
\hline & $\mathrm{Max}, \mu \mathrm{g} / \mathrm{kg}$ & 8903 & 8013 & 8292 & 8903 \\
\hline & 1st Quartile, $\mu \mathrm{g} / \mathrm{kg}$ & 1310 & 903 & 683.2 & 933 \\
\hline & 3rd Quartile, $\mu \mathrm{g} / \mathrm{kg}$ & 4258 & 3302 & 3166 & 3517 \\
\hline & Median, $\mu \mathrm{g} / \mathrm{kg}$ & 2690 & 1734 & 1637 & 1948 \\
\hline & Mean, $\mu \mathrm{g} / \mathrm{kg}$ & 3020 & 2253 & 2214 & 2496 \\
\hline & Standard deviation & 2057 & 1932 & 1959 & 2005 \\
\hline & Skewness & 0.9513 & 1.36 & 1.35 & 1.18 \\
\hline & Kurtosis excess & 3.506 & 4.42 & 4.66 & 4.01 \\
\hline \multirow[t]{10}{*}{$\mathrm{FB}_{2}$} & Number of points & 50 & 50 & 50 & 150 \\
\hline & Min, $\mu \mathrm{g} / \mathrm{kg}$ & 129 & 119 & 109 & 109 \\
\hline & $\operatorname{Max}, \mu g / \mathrm{kg}$ & 6115 & 5604 & 4711 & 6115 \\
\hline & 1st Quartile, $\mu \mathrm{g} / \mathrm{kg}$ & 637 & 402 & 320 & 430 \\
\hline & 3rd Quartile, $\mu \mathrm{g} / \mathrm{kg}$ & 1970 & 1552 & 1563 & 1660 \\
\hline & Median, $\mu \mathrm{g} / \mathrm{kg}$ & 1285 & 856 & 746 & 881 \\
\hline & Mean, $\mu \mathrm{g} / \mathrm{kg}$ & 1414 & 1193 & 1068 & 1225 \\
\hline & Standard deviation & 1116 & 1229 & 973 & 1113 \\
\hline & Skewness & 1.86 & 2.04 & 1.57 & 1.88 \\
\hline & Kurtosis excess & 7.89 & 6.95 & 5.79 & 7.25 \\
\hline \multirow[t]{8}{*}{ Fusarium infected kernels } & Number of points & 50 & 50 & 50 & 50 \\
\hline & Min. count & 0 & 0 & 0 & 0 \\
\hline & Max, count & 17 & 25 & 7 & 25 \\
\hline & Median count & 3 & 2 & 2 & 2 \\
\hline & Mean count & 3 & 2.5 & 2.44 & 2.65 \\
\hline & Standard deviation & 2.76 & 3.56 & 1.73 & 2.79 \\
\hline & Skewness & 2.95 & 5.35 & 0.46 & 4.57 \\
\hline & Kurtosis excess & 12.79 & 33.57 & -0.33 & 31.48 \\
\hline
\end{tabular}


Table 2. Descriptive statistics by layer of data collected after Box-Cox transformation

\begin{tabular}{llrrrr}
\hline Fumonisin & Statistic & Layer 1 & Layer 2 & Layer 3 & Combined \\
\hline $\mathrm{FB}_{1}$ & Number of points & 50 & 50 & 50 & 150 \\
& Min & 12.56 & 9.86 & 9.29 & 9.29 \\
& Max & 28.93 & 28.17 & 28.42 & 28.93 \\
& 17.37 & 15.71 & 14.41 & 15.82 \\
& 24.19 & 22.61 & 10.40 & 22.77 \\
& 3rd Quartile & 21.20 & 18.84 & 18.53 & 19.44 \\
Median & 20.76 & 18.72 & 18.43 & 19.30 \\
Mean & 4.14 & 4.61 & 4.96 & 4.67 \\
Standard deviation & 0.59 & 0.66 & 0.71 & 0.38 \\
Standard error of mean & -0.11 & 0.13 & 0.016 & -0.05 \\
Skewness & -0.72 & -0.70 & -0.85 & -0.76 \\
\hline Kurtosis excess & 50 & 50 & 50 & 150 \\
& Number of points & 6.08 & 5.95 & 5.82 & 5.82 \\
Min & 13.18 & 12.99 & 12.62 & 13.18 \\
& Max & 8.71 & 7.92 & 7.50 & 8.02 \\
& 10.84 & 10.44 & 10.40 & 10.51 \\
& 1 st Quartile & 9.01 & 9.26 & 9.00 & 9.31 \\
3rd Quartile & 9.69 & 9.15 & 8.99 & 9.27 \\
Median & 1.52 & 1.72 & 1.70 & 1.67 \\
Mean & 0.22 & 0.25 & 0.24 & 0.13 \\
Standard deviation & -0.31 & 0.24 & 0.065 & -0.018 \\
Standard error of mean & 0.058 & -0.46 & -0.97 & -0.64 \\
\hline Skewness & & & & \\
Kurtosis excess & &
\end{tabular}

The two-dimensional experimental semi-variograms (Figure 5) show that there is no clear spatial structure in any of the layers, so no empirical variogram was fitted. Layer 1 showed the smallest semi-variance values, followed by layer 2 and 3 . This is consistent with the standard deviation values shown in Table 2 . The increase in semi-variance values was more obvious for $\mathrm{FB}_{1}$ than $\mathrm{FB}_{2}$.

The results for the three-dimensional analysis showed that the semi-variance values increased with distance for both fumonisins (Figure 6). This pattern was clearer for $\mathrm{FB}_{1}$. The exponential model provided a better fit for the experimental semi-variogram of $\mathrm{FB}_{1}$ whilst the spherical was a better fit for $\mathrm{FB}_{2}$. The semi-variogram for $\mathrm{FB}_{1}$ showed a range of $1.83 \mathrm{~m}$, a sill of 24.23 and a nugget of 17.18. $\mathrm{FB}_{2}$ had a range of $0.62 \mathrm{~m}$, a sill of 2.71 and 0 nugget. The spatial autocorrelation was lost at closer distances for $\mathrm{FB}_{1}$ than for $\mathrm{FB}_{2}$.

The cross-variograms were pure nugget: none of the cross-variograms showed any significant spatial autocorrelation between the variables (Figures 7 and 8). This indicates that there is no spatial correlation between the two fumonisins or between the number of infected kernels and the fumonisin concentration.

\section{Discussion}

The results of the geostatistical analysis showed no evidence of spatial structure for fumonisins in two dimensions. This contrasts with Rivas et al. (2009a and 2009b) where DON in wheat, which is also produced by Fusarium spp. (F.culmorum, F.graminearum) presented spatial structure. There was some evidence of structure in the three dimensional semi-variogram for $\mathrm{FB}_{1}$. 
The random spatial pattern may be due to the nature of the mycotoxin, the way the grain was mixed and stored, the presence of spatial structure at a finer or coarser scale than the sampling grid or due to insufficient data points. There were only 50 points in each layer, whereas 100 are usually required (Webster and Oliver, 2000). The three-dimensional variogram contained 150 points, which may explain the appearance of some structure, but was limited by containing only three points in the third axis. If there is spatial structure at a finer scale than the sampling grid, it implies that practical sampling, which would normally be at a coarser scale, could treat the distribution as random.

Fumonisins $\mathrm{B}_{1}$ and $\mathrm{B}_{2}$ showed similar distribution patterns within each layer when visually inspected. The similarity was expected because both are produced by the same organisms. Few data are available on the ratio of $\mathrm{FB}_{2} / \mathrm{FB}_{1}$ and $\mathrm{FB}_{2}$ seems more prevalent than in a previous report (Battilani et al., 2008). However, the patterns of both fumonisins were not sufficiently correlated for the cross-variograms to detect it. Similarly, the cross-variogram did not show significant correlation between the number of Fusarium infected kernels and each of the fumonisins. Again this could be due to the limited number of samples. It may also be that the absence of infected kernels gives a low or zero mycotoxin concentration, but the presence of a large number of infected kernels does not necessarily correspond to large concentrations because of other factors.

Given the evidence found for spatial structure in two dimensions by Rivas Casado et al. (2009a) and the inconclusive results here, further research is needed into the presence of three-dimensional structure. This needs to consider the influence of several factors, including the type (barn or silo) and depth of the store, the method of loading, the average level of contamination and the effects of mixing as commodities are moved from stores to vehicles for transport and back into storage. If there was spatial autocorrelation it could be isotropic (the same in all directions), or anisotropic if the vertical properties were different from the horizontal ones. There might also be vertical trends due to relationships between disease and grain size. The objective should be the development of practical sampling plans for monitoring.

The arrangement of the sampling planes in this study was constrained by the practical problems of sampling from a deep bed of maize and the available time and resources. The ideal protocol for such studies in future would sample without disturbance from as many horizontal planes as the depth allowed, and would have a minimum of $8 \times 8$ points, preferably $10 \times 10$, in each plane to give a rectilinear grid in three dimensions. Clearly equipment would have to be designed to facilitate this. Nested sampling designs could be implemented to characterise the spatial variability of the mycotoxins at different scales.

\section{Conclusions}

This paper presents the first analysis of the spatial structure of a three dimensional data set of fumonisin $B_{1}$ and $B_{2}$. There was no clear evidence of spatial structure in either the twodimensional or three-dimensional analyses. The number of infected kernels was not a good indicator for the prediction of fumonisin concentration and there was no spatial correlation between the concentrations of the two fumonisins.

\section{Acknowledgements}


This research was funded by the UK Food Standards Agency Project CO3055.

\section{References}

Barnes R.J. 1991. The variogram sill and the sample variance. Mathematical Geology 23(4): 673-678

Battilani P., Barbano C., Rossi V., Bertuzzi T., Pietri A. 2006. Spatial distribution of ochratoxin A in vineyard and sampling design to assess must contamination. Journal of Food Protection, 69 (4), 884-890.

Battilani P., Pietri A., Barbano C., Scandolara A., Bertuzzi T. and Marocco A. 2008. Logistic Regression Modeling of Cropping Systems To Predict Fumonisin Contamination in Maize. Journal of Agricultural and Food Chemistry, 56, 10433-10438.

Burgess, L.W., Summerell, B.A., Bullock, S., Gott, K.P. and Backhouse, D. (1994) Laboratory Manual for Fusarium Research, 3rd Edition. Department of Crop Science, University of Sydney/Royal Botanic Gardens, 134 p.

Chehata M., Jasinski D., Monteith M.C., Samuels W.B. 2007. Mapping three-dimensional water quality data in the Chesapeake Bay using geostatistics. JAWRA 43(3): 813-828.

Coker R.D., Nagler M.J., Blunden G., Sharkey A.J., Defize P.R., Derksan G.B., Whitaker T.B. 1995. Design of sampling plan for mycotoxins in foods and feeds. Natural Toxins 3: 257-264.

Desjardins A.E., Plattner R.D., Lu M., Claflin L.W. 1998. Distribution of fumonisins in maize ears infected with strains of Fusarium moniliforme that differ in fumonisin production. Plant Disease 82(8): 953-958.

European Commission. 2006a. Commission Regulation 401/2006 of 23 February 2006. Laying down the methods of sampling and analysis for the official control of the levels of mycotoxins in foodstuffs. Official Journal of the European Union. L70: 12-34.

European Commission. 2006b. Commission Regulation 1881/2006 of 19 December 2006. Setting maximum levels for certain contaminants in foodstuffs. Official Journal of the European Union. L364: 5-24.

European Commission. 2007. Commission Regulation 1126/2007 of 28 September 2007 amending Regulation 1881/2006 setting maximum levels for certain contaminants in foodstuffs as regards Fusarium toxins in maize and maize products. Official Journal of the European Union. L255: 14-17.

Falivene O., Cabrera L., Saez A. 2007. Large to intermediate-scale aquifer heterogeneity in fine-grain dominated alluvial fans (Cenozic As Pontes Basin, northwestern Spain): insight based on three-dimensional geostatistical reconstruction. Hydrogeology Journal 15: 861-876.

Jewers K., Bradburn N., Sharkey A.J. 1988. Aflatoxin distribution studies on a 4 tonne batch of maize. International Biodeterioration 24(4-5): 393-398.

Johansson A.S., Whitaker T.B., Hagler W.M.J.R., Bowman T., Slate A.B., Payne G. 2006. Predicting aflatoxin and fumonisin in shelled corn lots using poor-quality grade components. Journal of AOAC International 89: 433-440.

Maanen A., Xu X.M. 2003. Modelling plant disease epidemics. European Journal of Plant Pathology 109: 669-682. 
Macarthur R., Macdonald S., Brereton P., Murray A. 2006. Statistical modelling as an aid to the design of retail sampling plans for mycotoxins in food. Food Additives and Contaminants 23 (1): 84-92.

Nielsen D.R., Wendroth O. 2003. Spatial and temporal statistics: sampling field soils and their vegetation. GeoEcology textbook. Reiskirchen: Catena Verlag.

Reid L.M., Mather D.E., Hamilton R.I. 1996. Distribution of Deoxynivalenol in Fusarium graminearum-Infected Maize Ears. Phytopathology 86 (1): 110-114.

Rekah Y., Shtienberg D., Katan J. 1999. Spatial distribution and temporal development of fusarium crown and root rot of tomato and pathogen dissemination in field soil. Phytopathology. 89(9): 831-839.

Rivas Casado M., Parsons D.J., Weightman R.M., Magan N., Origgi S. 2009a. Geostatistical analysis of the spatial distribution of mycotoxin concentration in bulk cereals. Food Additives and Contaminants 26 (6): 867-873.

Rivas Casado M., Parsons D.J., Weightman R.M., Magan N., Origgi S. 2009b. Modelling the spatial distribution of mycotoxin concentration in bulk commodities to design effective and efficient sampling strategies. Food Additives and Contaminants 26 (9): 1298 - ?

Schatzki T.F. 1995a. Distribution of aflatoxin in pistachios. 1. Lot distributions. Journal of Agricultural and Food Chemistry 43(6): 1561-1565.

Schatzki T.F. 1995b. Distribution of aflatoxin in Pistachios. 2. Distribution in freshly harvested pistachios. Journal of Agricultural and Food Chemistry 43(6): 1566-1569.

Stavropoulou M., Exadaktylos G., Saratsis G. 2007. A combined three-dimensional geological-geostatistical-numerical model of underground excavations in rock. Rock Mechanics and Rock Engineering 40 (3): 213-243.

Stroka J., Spanjer M., Buechler S., Barel S., Kos G., Anklam E. 2004. Novel sampling methods for the analysis of mycotoxins and the combination with spectroscopic methods for the rapid evaluation of deoxynivalenol contamination. Toxicology Letters 153(1): 99-107.

Uhlig S., Torp M., Jarp J., Parich A., Gutleb A.C., Krska R. 2004. Moniliformin in Norwegian grain. Food Additves and Contaminants 21(6): 598-606.

Visconti, A., Solfrizzo, M., Girolamo, A. de. 2001. Determination of fumonisins $\mathrm{B}_{1}$ and $\mathrm{B}_{2}$ in corn and corn flakes by liquid chromatography with immunoaffinity column cleanup: collaborative study. Journal of AOAC International 84 (6): 1828-1837.

Webster R., Oliver M.A. 2000. Geostatistics for environmental scientists. Chichester: John Wiley and Sons, Ltd.

Whitaker T.B. 2003. Standardisation of mycotoxin sampling procedures: an urgent necessity. Food Control 14 (4): 233-237.

Whitaker T.B. 2006. Sampling foods for mycotoxins. Food Additives and Contaminants 23(1): 50-61. 


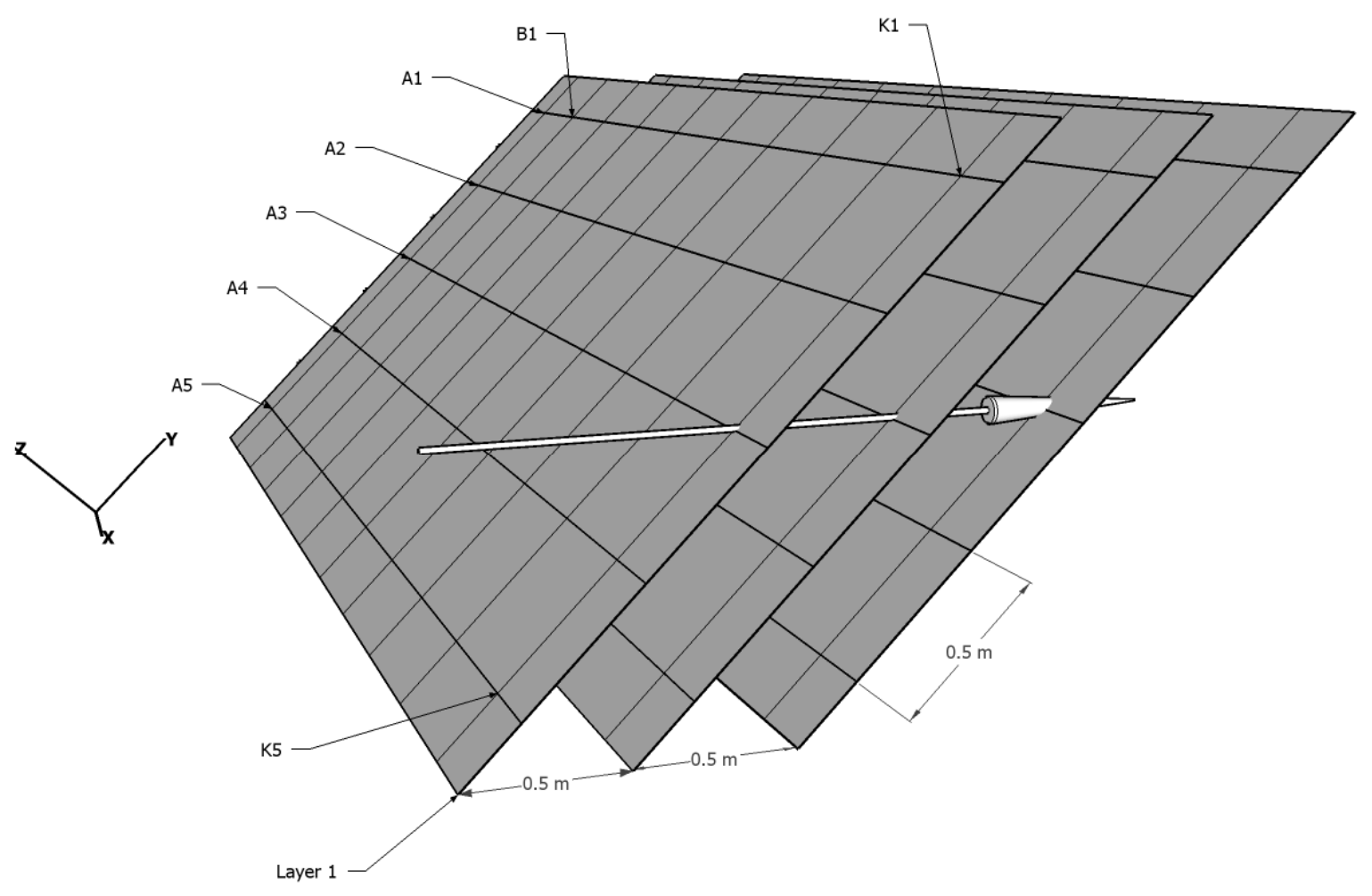

Figure 1. Schematic of the sampling locations and probe (not to scale)

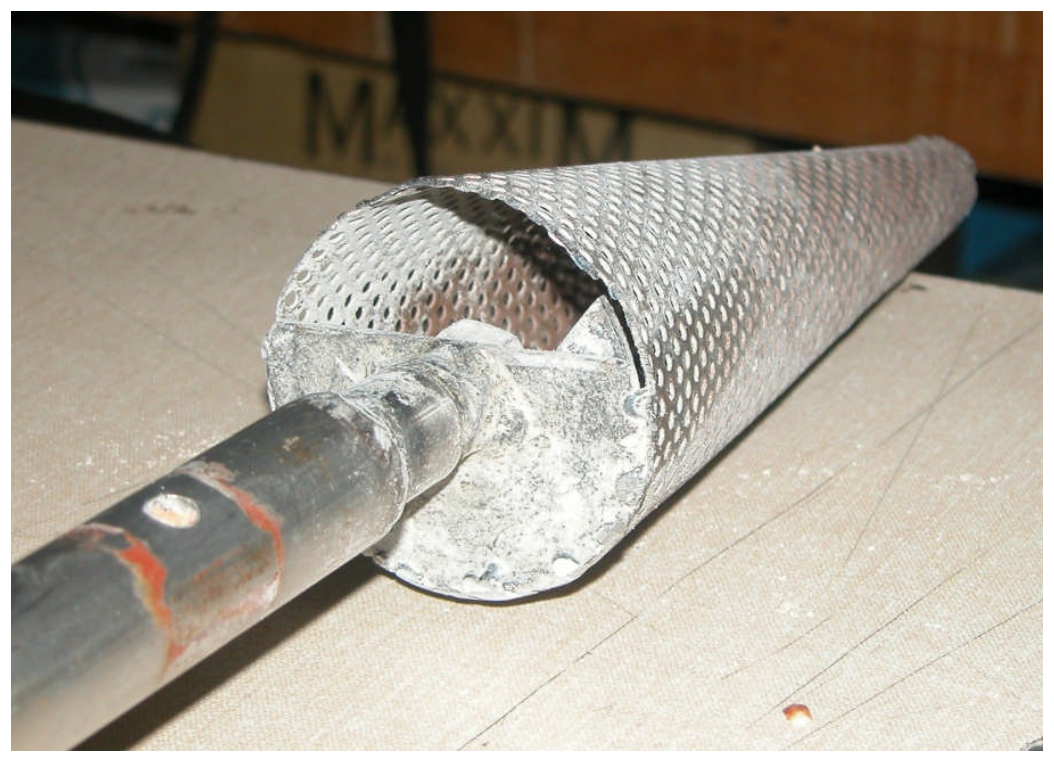

Figure 2. Detail of the chamber on the sampling probe in the open position 


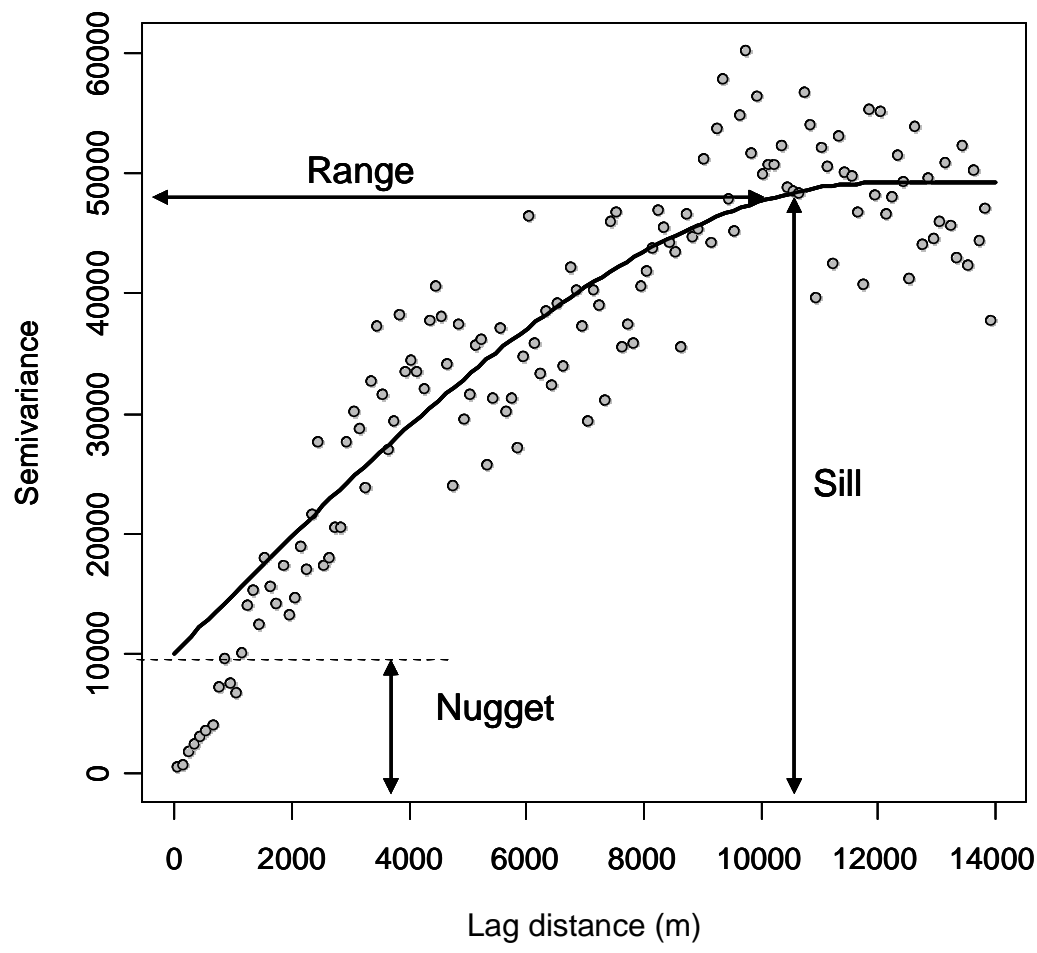

Figure 3. Typical experimental (points) and empirical (line) variograms showing the semi-variogram parameters 
FB1 layer 1

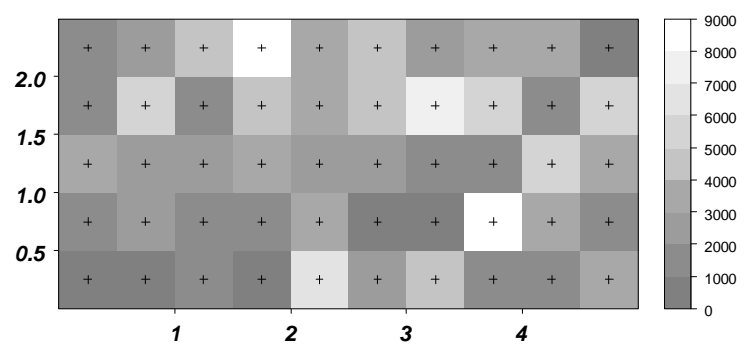

FB1 layer 2

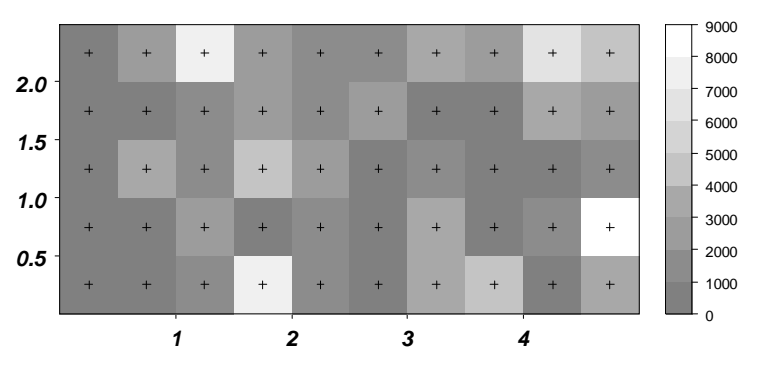

FB1 layer 3

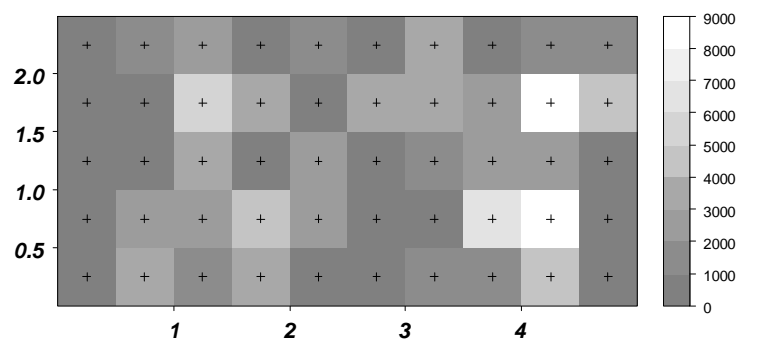

FB2 layer 1
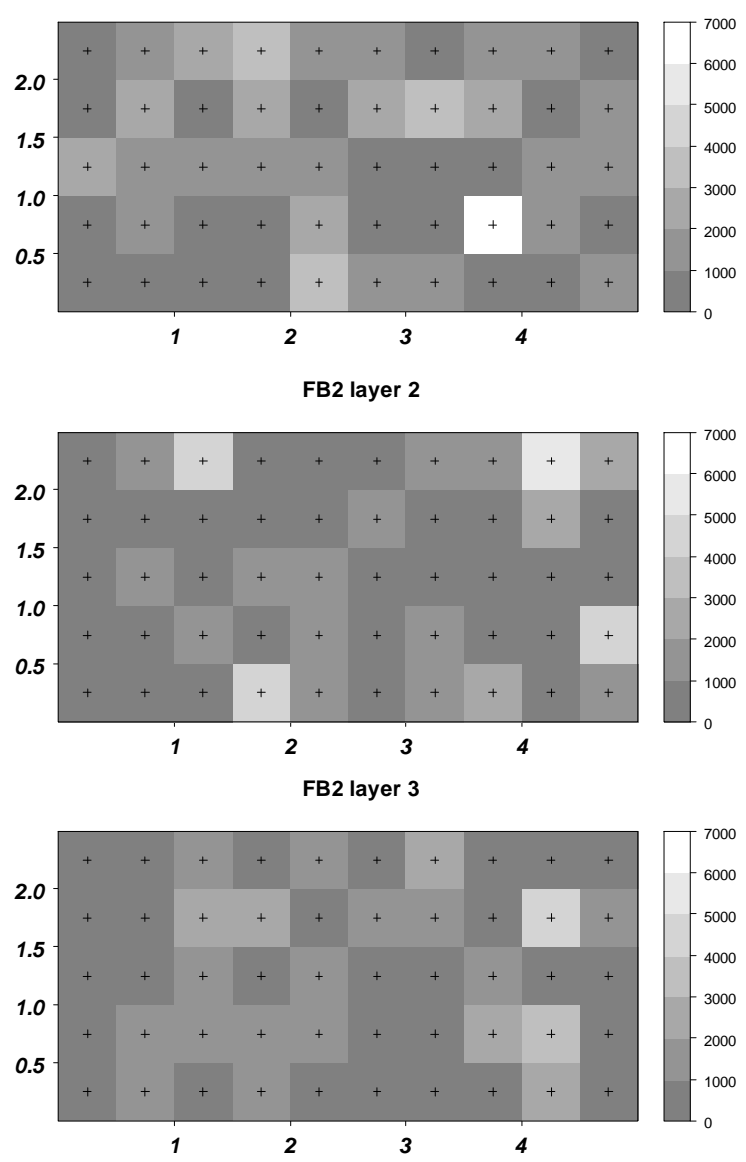

Figure 4. Grid-cell plots showing the spatial distribution of fumonisin $B_{1}$ and $B_{2}$ per sampled layer. Concentrations are given in $\mu \mathrm{g} / \mathrm{kg}$. 

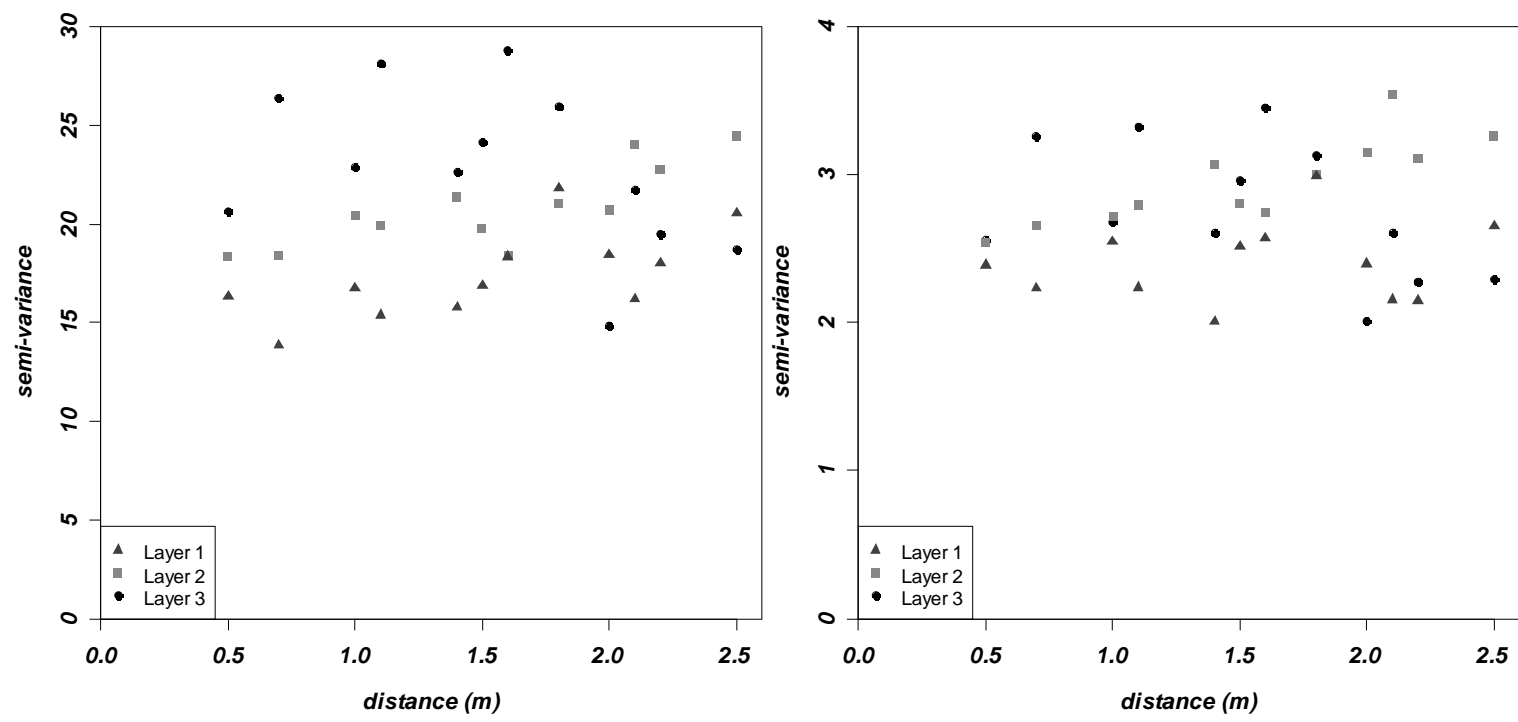

Figure 5. Two dimensional semi-variogram for each of the layers for fumonisin B1 (left) and B2 (right)
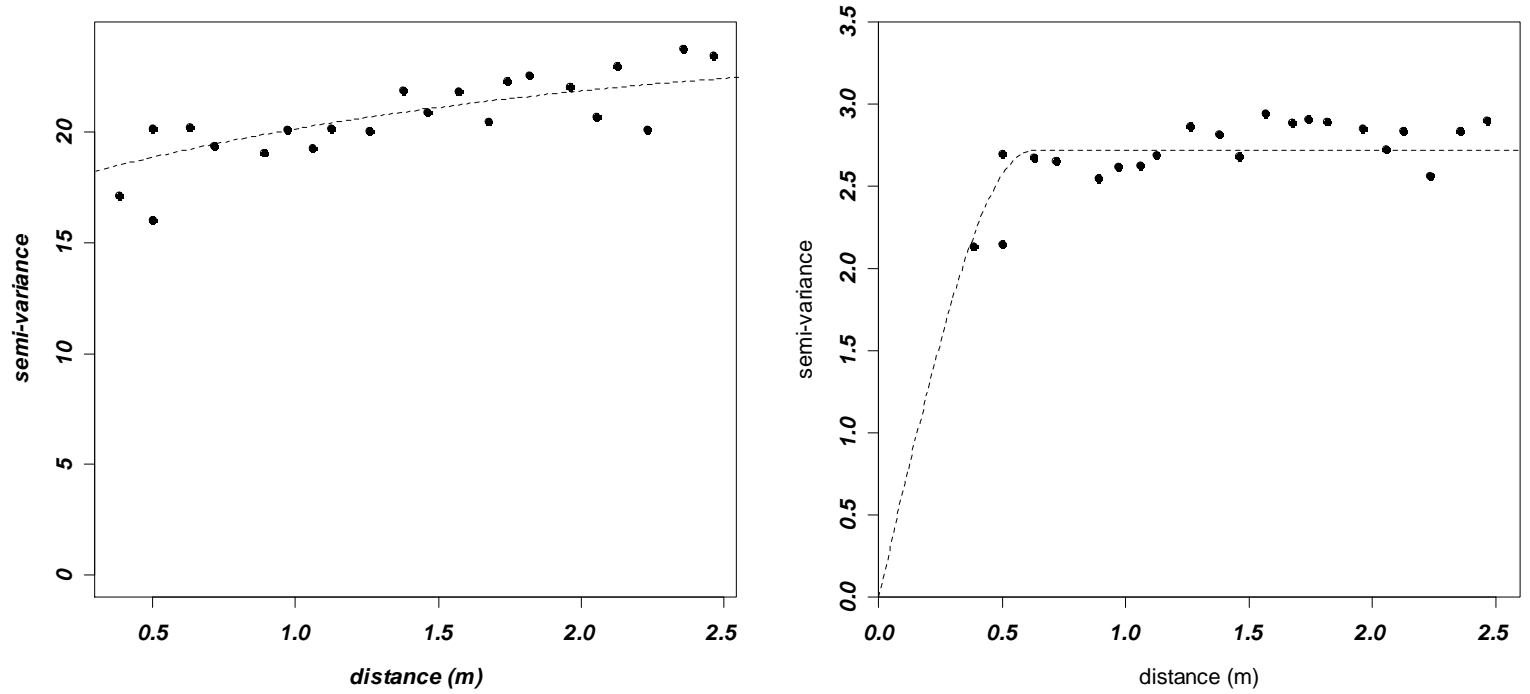

Figure 6. Three dimensional semi-variogram for fumonisin B1 (left) and B2 (right) 


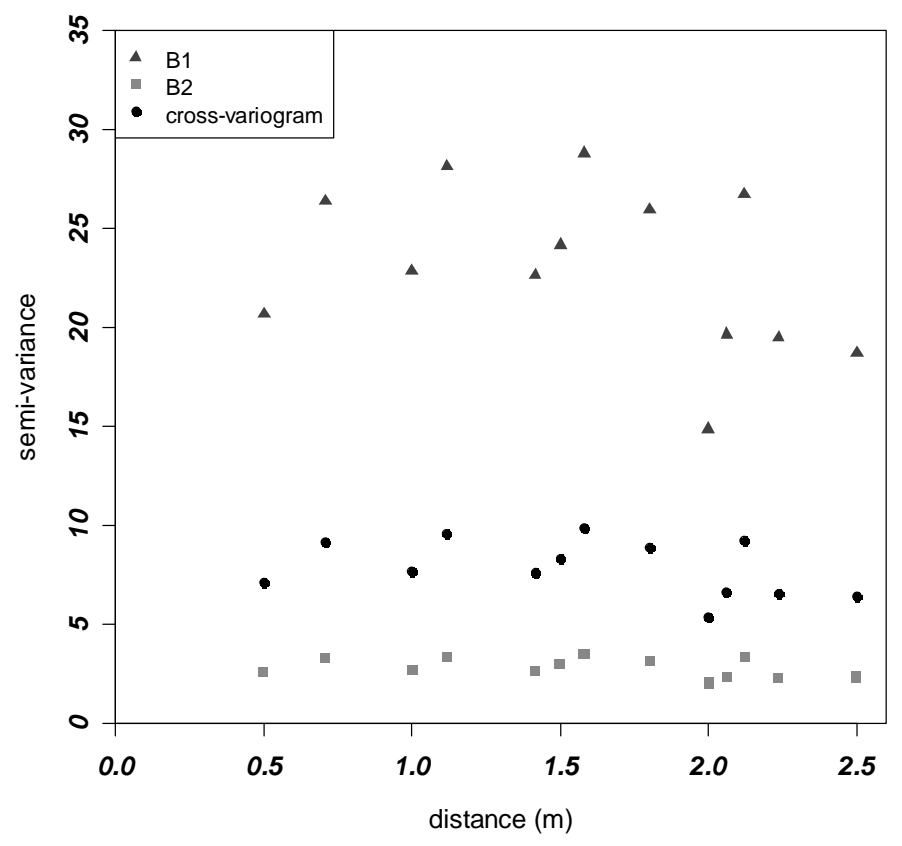

Figure 7. Semi-variograms for fumonisin $\mathrm{B} 1$ and $\mathrm{B} 2$ and the cross-variogram for the two fumonisins in layer 3.

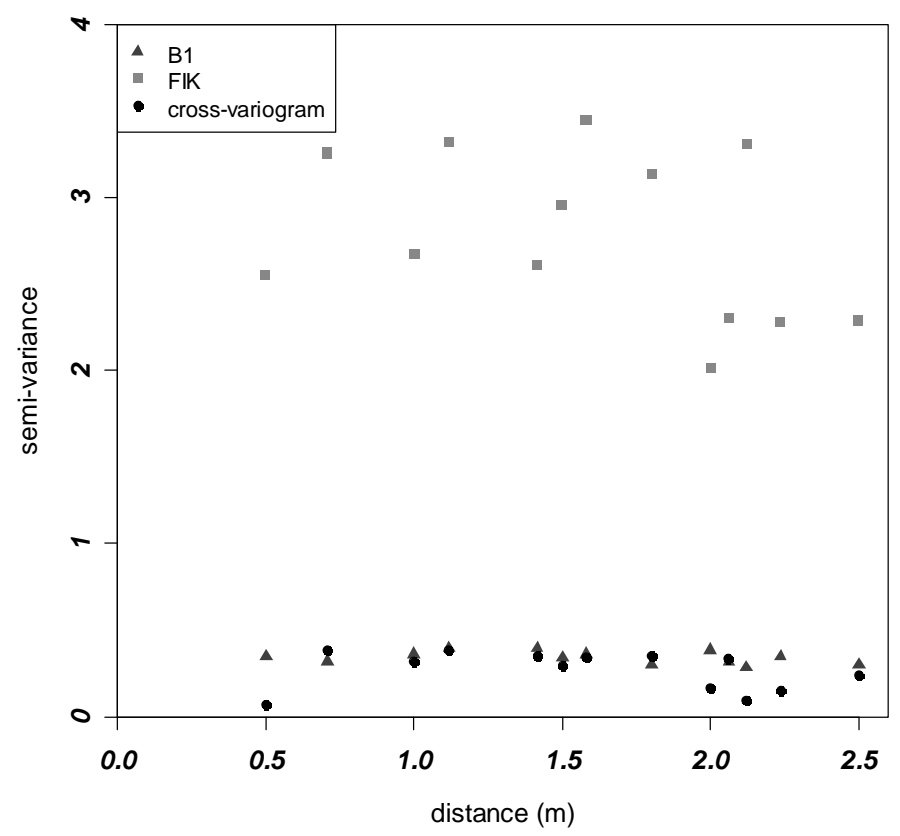

Figure 8. Semi-variograms for fumonisin B1 and number of infected kernels (labelled B3) and their cross-variogram in layer 3. 